\title{
Comparative genomics of Glandirana rugosa using unsupervised Al reveals a high CG frequency
}

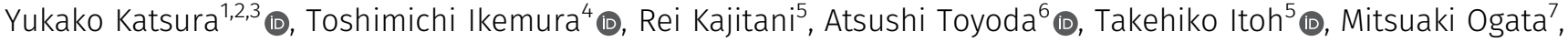 \\ Ikuo Miura ${ }^{2}$, Kennosuke Wada ${ }^{4}$, Yoshiko Wada ${ }^{4}$, Yoko Satta ${ }^{3}$
}

\begin{abstract}
The Japanese wrinkled frog (Glandirana rugosa) is unique in having both XX-XY and ZZ-ZW types of sex chromosomes within the species. The genome sequencing and comparative genomics with other frogs should be important to understand mechanisms of turnover of sex chromosomes within one species or during a short period. In this study, we analyzed the newly sequenced genome of $G$. rugosa using a batch-learning self-organizing map which is unsupervised artificial intelligence for oligonucleotide compositions. To clarify genome characteristics of G. rugosa, we compared its short oligonucleotide compositions in all 1-Mb genomic fragments with those of other six frog species (Pyxicephalus adspersus, Rhinella marina, Spea multiplicata, Leptobrachium leishanense, Xenopus laevis, and Xenopus tropicalis). In G. rugosa, we found an Mb-level large size of repeat sequences having a high identity with the $W$ chromosome of the African bullfrog ( $P$. adspersus). Our study concluded that $G$. rugosa has unique genome characteristics with a high CG frequency, and its genome is assumed to heterochromatinize a large size of genome via methylataion of CG.
\end{abstract}

DOI 10.26508/lsa.202000905 | Received 9 September 2020 | Revised 16 February 2021 | Accepted 17 February 2021 | Published online 12 March 2021

\section{Introduction}

The sex determination system (XX-XY or ZZ-ZW) is known to change often during evolution of organisms, and the systems in amphibians and fishes have changed at a higher rate than that in mammals and birds (Bachtrog et al, 2014). In particular, the Japanese wrinkled frog (Glandirana rugosa) is unique in having both XX-XY and ZZ-ZW systems within the species (Miura, 2007, 2017), indicating that alteration of the systems is still ongoing (Ogata et al, 2018); for details of the sex determination system of G. rugosa, see the Discussion section. To clarify its genome characteristics, we have decoded the genome sequence of $\mathrm{G}$. rugosa which is a diploid species, but whose assembled genome size $(7.08 \mathrm{~Gb})$ is larger than that of the tetraploid Xenopus laevis (2.7 Gb; Session et al, 2016; Li et al, 2019) and $\sim 4.5$ times larger than that of the evolutionarily related diploid Pyxicephalus adspersus (1.56 Gb; Denton et al, 2018 Preprint). The large $G$. rugosa genome is due not to whole genome duplication, but probably because of explosive proliferation of transposons or partial genome duplications. In a large genome, it is conceivable that expression in a wide area of the genome is suppressed, and comparisons with the small or different size of genomes should yield knowledge about the molecular mechanisms of the suppression in a wide genomic range. A research project aimed at complete genome sequencing of G. rugosa, including an advanced assembly, gene annotations, and chromosomal attributions, is still underway, but the present study focused on the analysis of speciesspecific genomic characteristics and searched for possible structures involved in sex chromosomes.

To understand the G. rugosa genome characteristics by comparing its large genome with other frog ones, the present study introduces a new strategy that uses unsupervised artificial intelligence (AI) because Al (machine learning) has become an essential technology for efficient data mining from large and complex data. Notably, unsupervised Al can discover new knowledge without particular models or prior knowledge and is highly desirable for unveiling characteristics hidden in the data; this is a data-driven research based on findings by the unsupervised Al. Specifically, comparative genomics was performed by the batch-learning selforganizing map (BLSOM) using short oligonucleotides (Abe et al, 2003). The short oligonucleotide composition is unique characteristics to each species and is often described as a "genome signature" meaning a characteristic frequency of oligonucleotides (Karlin, 1998); importantly, even if the genome is fragmented (e.g., to $100 \mathrm{~kb}$ ), most of the fragments have a similar oligonucleotide composition. The genome signature of many species has been visualized easily by the BLSOM (Abe et al, 2005, 2006; Nakao et al, 2013). Using the oligonucleotide BLSOM, we previously analyzed the human genome and found a large $\mathrm{Mb}$-level structure consisting of

\footnotetext{
${ }^{1}$ Primate Research Institute, Kyoto University, Inuyama-shi, Japan ${ }^{2}$ Amphibian Research Center, Hiroshima University, Hiroshima-shi, Japan ${ }^{3}$ Department of Evolutionary Studies of Biosystems, School of Advanced Sciences, The Graduate University For Advanced Studies (SOKENDAI), Shonankokuraimura, Hayama-machi, Japan ${ }^{4}$ Department of Bioscience, Nagahama Institute of Bio-Science and Technology, Nagahama-shi, Japan ${ }^{5}$ Department of Life Science and Technology, School of Life Science and Technology, Tokyo Institute of Technology, Tokyo-to, Japan ${ }^{6}$ Department of Genomics and Evolutionary Biology, National Institute of Genetics, Mishima-shi, Japan ${ }^{7}$ Preservation and Research Center, Yokohama, Japan
} 
repetitive sequences rich in $\mathrm{CG}$ (the $\mathrm{Mb}$-level $\mathrm{CpG}$ island) in centromeric and pericentromeric heterochromatin, as well as in subtelomeric regions (Wada et al, 2015, 2020). In this study, we also found $\mathrm{Mb}$-level large $\mathrm{CpG}$ islands on frog genomes by comparative genomics using seven species (G. rugosa, P. adspersus, Rhinella marina, Spea multiplicata, Leptobrachium leishanense, X. laevis, and Xenopus tropicalis) and showed that $G$. rugosa had genome characteristics with a high CG frequency.

\section{Results}

\section{BLSOM analysis}

We analyzed the short oligonucleotide composition in genomes of seven frogs, including G. rugosa using the BLSOM (Fig 1); the current status of genome sequencing of $G$. rugosa is explained in the Materials and Methods section. Because the oligonucleotide composition inevitably depends on mononucleotide compositions, we first constructed a BLSOM with the mononucleotide composition of all 100-kb sequences derived from the seven genomes (Figs
1A and S1). The total number of nodes (grid points) was set to 1/10 of the total number of sequences $(144,623)$; each node thus has an average of 10 sequences. In Fig $1 \mathrm{Ai}$, grid points containing sequences of a single species are colored to indicate each species, and grid points containing sequences of multiple species are displayed in black. Most points are black, showing that the sequences are not separated accurately by species. Next, when sequences of a single species occupy more than $50 \%$ at a grid point, the color indicating that species is given (Fig 1Aii), and this shows that the mononucleotide composition differs among species even when fragmented to $100 \mathrm{~kb}$; G. rugosa and L. leishanense with high genome $\mathrm{G}+\mathrm{C} \%$ among the frog genomes ( $44.5 \%$ and $43.4 \%$, respectively) are located on the left side in the map (Fig 1Aii), but their sequences are intermingled there; $X$. laevis and $P$. adspersus with low genome $\mathrm{G}+\mathrm{C} \%$ (38.5\% and $37.9 \%$, respectively) are on the right side.

Each genome consists of double strands, but only one-strand sequence is registered by The International Nucleotide Sequence Database Collaboration; in the case of a scaffold sequence, there is arbitrariness as to which strand is registered. Furthermore, when general features of genomic sequences of one species (e.g., genome

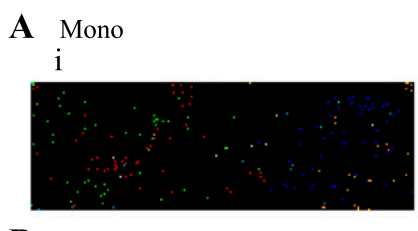

ii

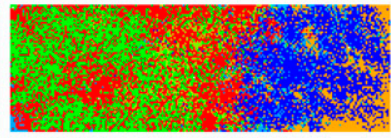

B Di

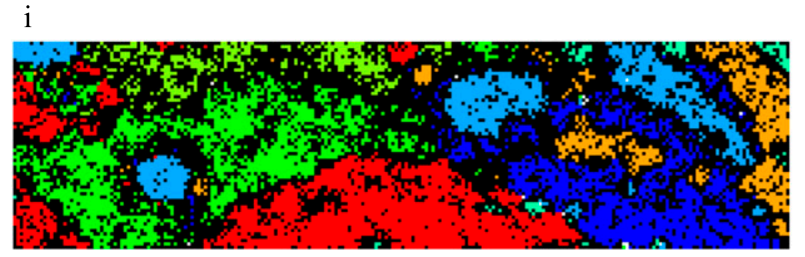

ii

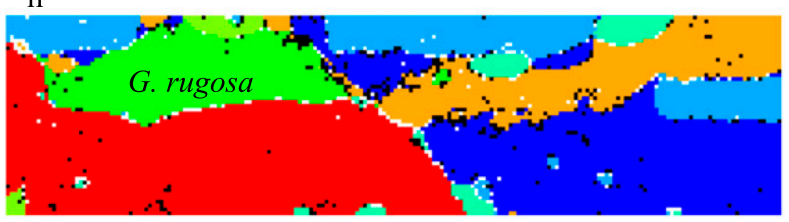

L. leishanense (†), P. adspersus (), R. marina (), G. rugosa (), S. multiplicata ()$, X$. tropicalis () and X. laevis (ロ).

C

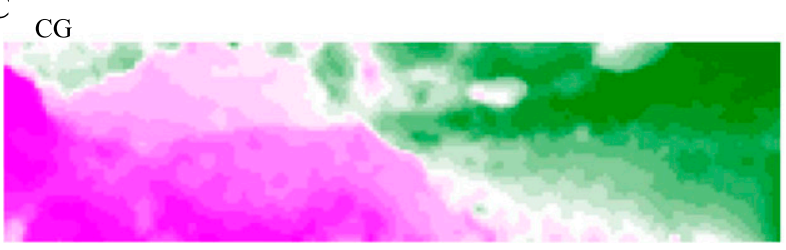

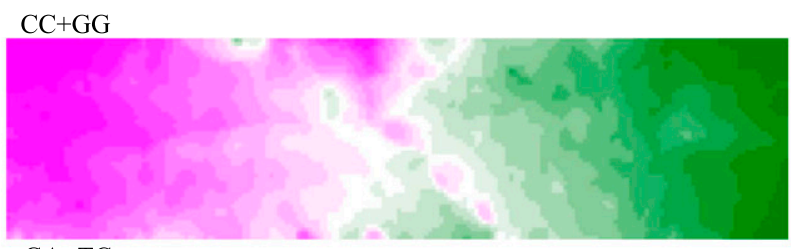
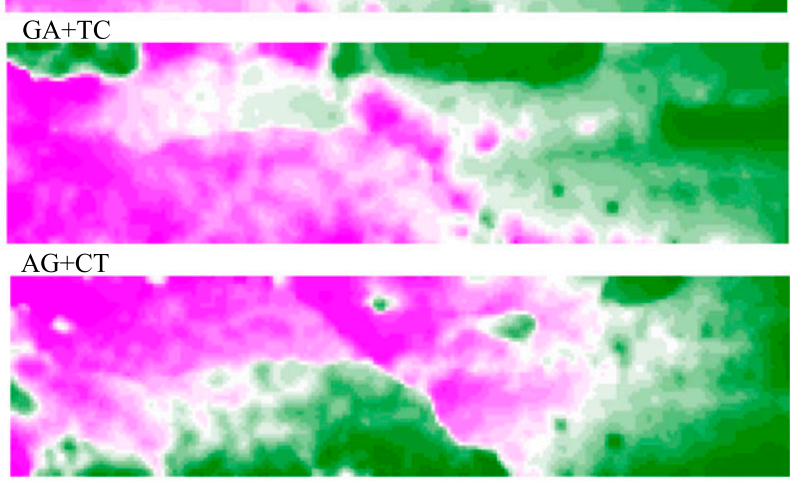

D

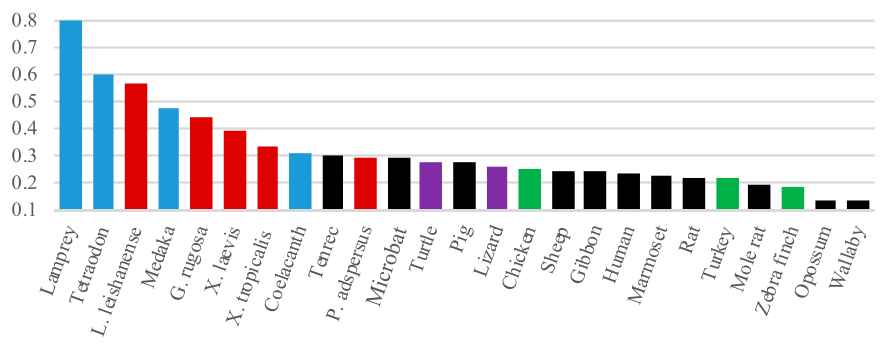

Figure 1. Batch-learning self-organizing maps (BLSOMs) and normalized CG levels.

(Ai, Aii) Mononucleotide (Mono) BLSOMs for 100-kb frog sequences. Nodes containing sequences from more than one species are indicated in black, and those containing sequences only from one species are indicated in color to distinguish species; nodes that do not contain sequnces after machine learning were left as blank (white) (Ai). When sequences of a single species occupy more than $50 \%$ at a node, the color indicating that species is given (Aii). (Bi, Bii) Dinucleotide (Di) BLSOMs for $100-\mathrm{kb}$ sequences and 1-Mb sequences sliding with a 100-kb step, respectively. Nodes are marked as described in Ai. (C) The contribution level of each dinucleotide to each node is visualized by a color: pink (high), white (moderate), and green (low); results of all dinucleotides are presented in Fig S2. (D) Normalized CG levels of 25 vertebrates are arranged in the descending order: fishes (blue), frogs (reddish brown), reptiles (violet) and mammals (black). 
signature) are considered, there is little meaning in distinguishing complementary sequences. Therefore, a pair of complementary oligonucleotides are summed as a group in the present study; complementary oligonucleotides such as AA and TT are not distinguished, and their occurrences are summed (Abe et al, 2005).

Fig 1Bi shows a BLSOM for the composition of the degenerate sets of dinucleotides under the condition that an average of 10 sequences belongs to each node. Even though species information is not given during the machine learning, a large portion of $100-\mathrm{kb}$ sequences have clustered (self-organized) in the territories of each species and thus colored. Next, Fig 1Bii shows a BLSOM with the dinucleotide composition in all 1-Mb sequences sliding with 100$\mathrm{kb}$ width. Whereas the average number of sequences belonging to one node is almost the same as that in Fig 1Bi (10 sequences per node), separation into the species-specific colored territories becomes far clearer than that of the 100-kb BLSOM, making it easier to detect species-specific characteristics, such as genome signature. The subsequent BLSOM analyses, therefore, focus on the $1-\mathrm{Mb}$ sequences sliding with $100-\mathrm{kb}$ width. On the map of Fig 1Bii, L. leishanense (3.56 Gb; Li et al, 2019) and G. rugosa, which have particularly large assembled genomes, form their own large territories (red and green, respectively), but X. tropicalis (1.44 Gb; Hellsten et al, 2010) and P. adspersus (1.56 Gb; Denton et al, 2018 Preprint), which have small ones, form multiple territories rather than one large territory. In this study we use the assembled genome or total genome sequence length to compare their genome sizes because a genome size based on nuclear DNA content is not investigated or published yet in some frogs including $G$. rugosa. In the case of frogs ( $R$. marina and S. multiplicata) whose genome is only partially assembled, tiny territories are scattered often within a large territory of other species (Figs 1Bii and S1).

\section{Oligonucleotides prominently contributing to species-dependent separations}

The BLSOM is an explainable Al and can provide reasons for why a species-dependent separation (self-organization) has occurred. Fig $1 \mathrm{C}$ shows four examples of dinucleotides for the BLSOM listed in Fig $1 \mathrm{Bii}$; by focusing on vectorial data (i.e., dinucleotide frequencies) representing each grid point, the grid points are sorted in the descending order of their frequency for each dinucleotide. The descending order of grid points is then represented as a heat map for each dinucleotide; the higher rank points are displayed in pink, the middle ranks in white, and the lower ranks in green (Kanaya et al, 2001); results for all dinucleotides are listed in Fig S2. When searching the heat map pattern that reflects the speciesdependent separation, CG shows a good match with the separation (Fig 1C). Specifically, CG is characteristically higher (pink) in the territory of L. leishanense and G. rugosa than in others (green) (Fig 1C). The frequency of other dinucleotides does not show a clear match with species borders of territories; for example, $C C+G G$ is generally higher on the left side, but does not agree well with the species territories (Fig 1C). Notably, the separation of the dinucleotide BLSOM does not simply reflect an additive effect of the mononucleotide composition; for example, the frequency of GA+TC and $A G+C T$, which have the same mononucleotide composition, differs among species (Fig 1C).

\section{Comparison of CG occurrence among species}

The basic strategy of this data-driven investigation is to leave knowledge discovery to the unsupervised $\mathrm{Al}$ and, based on the obtained knowledge, to conduct more detailed analyses. It is of interest that CG occurs more frequently in $G$. rugosa and $L$. leishanense than in other species (Fig 1C). Methylation at C in CG is a typical epigenetic modification, and the methylated $C$ induces histone deacetylation, subsequent chromatin condensation, and heterochromatinization (Klose et al, 2005; Bogdanovic \& Veenstra, 2009). Hence, we conduct the detailed analyses of this biologically important dinucleotide in the frog genomes.

Because methyl-CG tends to mutate to TG/CA, CG is known to occur at a low frequency in vertebrate genomes: that is, CG suppression (Law \& Jacobsen, 2010). One index of the CG suppression, which excludes influences of mononucleotide compositions, is the odds ratio of CG which is obtained by dividing the observed occurrence (Obs) of CG by its expected value (Exp) calculated from the mononucleotide composition. Colored bars in Fig 1D show the Obs/ Exp values for genomes of 25 vertebrates covering a wide phylogenetic range (those for 45 vertebrates are presented in Fig S3); frog genomes with total reported contig sequences $(>1 \mathrm{Mb})>1 \mathrm{~Gb}$ are included. The CG suppression is evident in mammals (black), and their Obs/Exp values are distributed in a narrow range ( 0.2-0.3) except for the opossum and wallaby values (<0.2) (Fig 1D). In contrast, the Obs/Exp values for fishes (blue) vary considerably (0.7-0.3); for example, coelacanth is evolutionarily close to tetrapods, and the value for coelacanth is at the same level as that for tetrapods (Iwasaki et al, 2014). The Obs/Exp values for frogs (reddish brown) also vary considerably; the highest is 0.57 for L. leishanense, and G. rugosa is the second highest at 0.45 , but P. adspersus is 0.30 which corresponds to the reptile (violet) and mammal levels.

\section{CG distribution on each chromosome}

Fig 1Bii analyzed the dinucleotide composition in 1-Mb windows sliding with a 100-kb step, and using the same data set, we next plotted the CG composition (\%) along chromosomes of four frogs whose genome sequences have been assembled into each chromosome. Fig 2A-D shows the pattern of the CG composition on six chromosomes for each frog including the W chromosome (chrW) in $P$. adspersus. The basal level on the P. adspersus chromosomes is approximately half of the level for the L. leishanense chromosomes. In most cases, a distinct increase in CG toward both ends of each chromosome is observed, and the highest peak is generally located near the end. This is particularly obvious for $P$. adspersus and $X$. tropicalis, which have the low CG frequency throughout the whole genome. In Fig 2, the binomial approximation is shown as a reddish brown dashed line. As mentioned above, chrW of $P$. adspersus is an evident exception (Fig 2A-D); whereas the chrW sequences used here do not include the pseudoautosomal region homologous to chrZ, there was no an increase at either end of chrw, but instead the whole region has the high CG.

Although lower than the prominent CG peaks at both ends of chromosomes, internal peaks are observed. This is particularly 
A P. adspersus
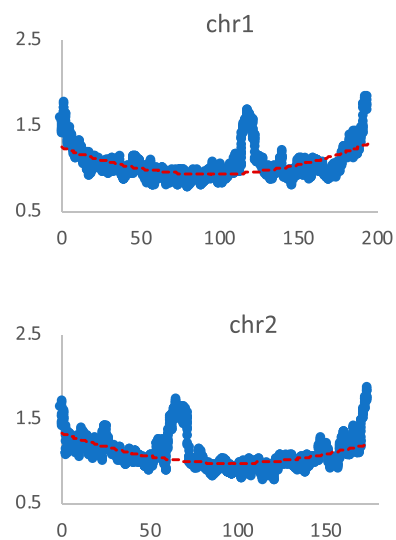

chr3

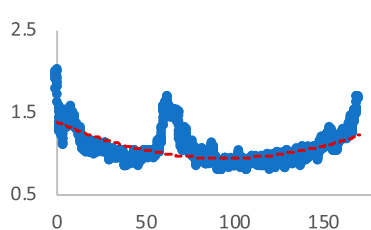

2.5

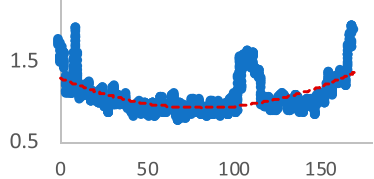

chr5
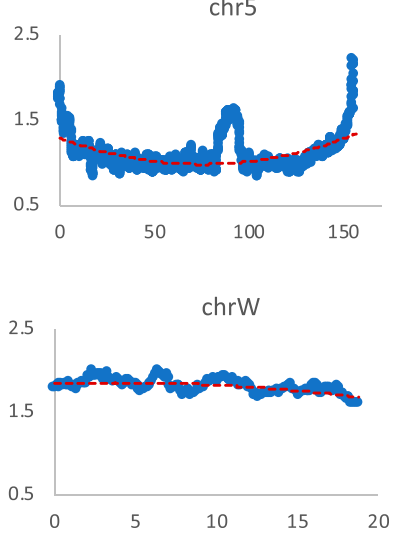

B X. tropicalis

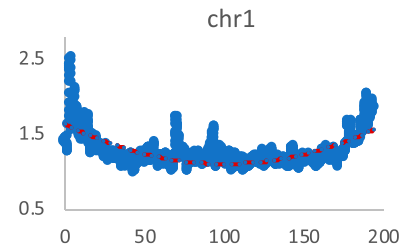

chr2

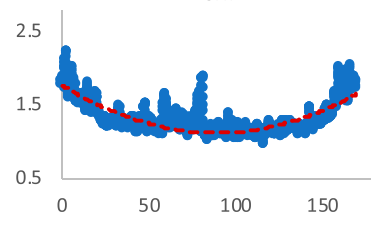

chr3

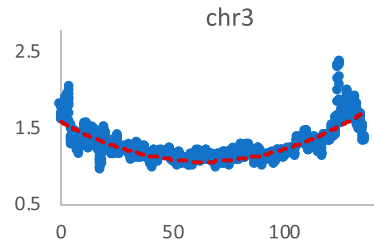

chr4
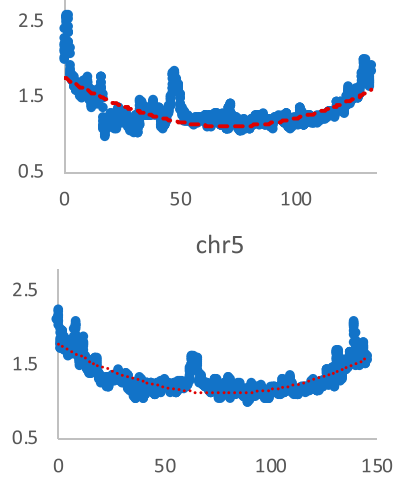

chr6

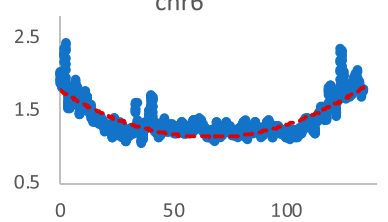

C X. laevis

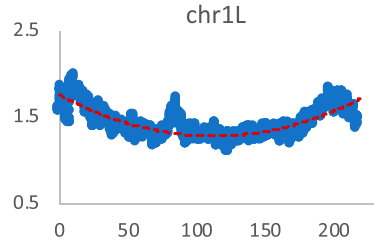

chr2L
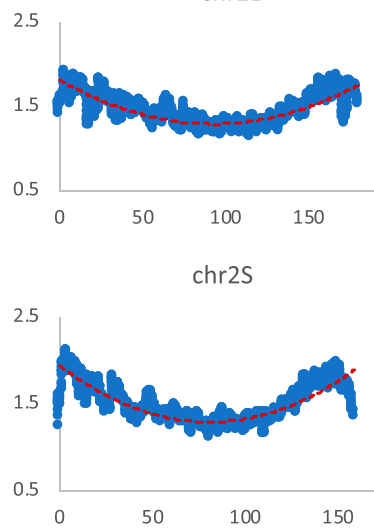

chr3L

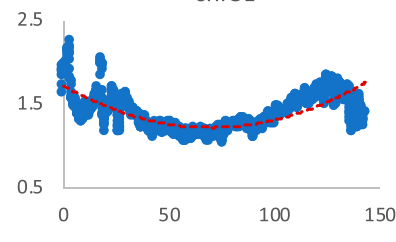

chr3s

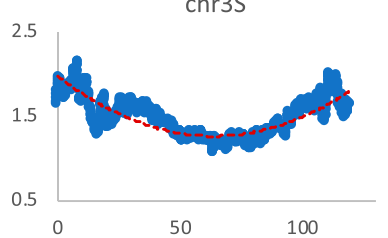

chr4L

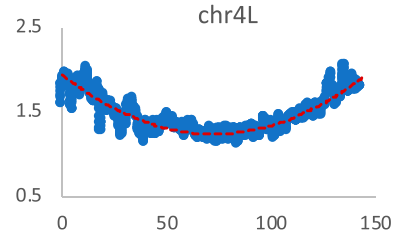

D L. leishanense

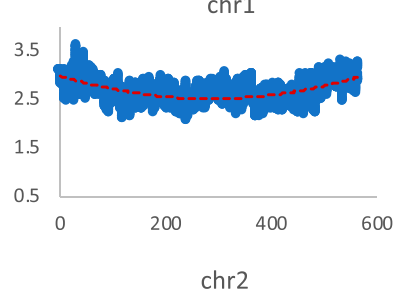

chr2

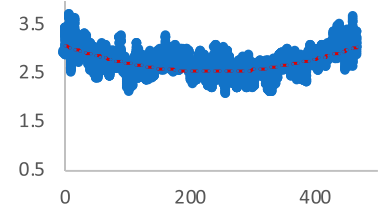

chr3

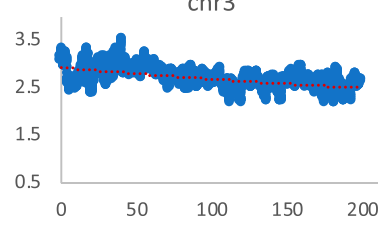

chr4

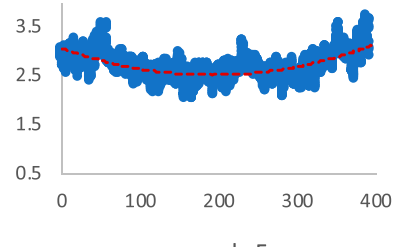

chr5
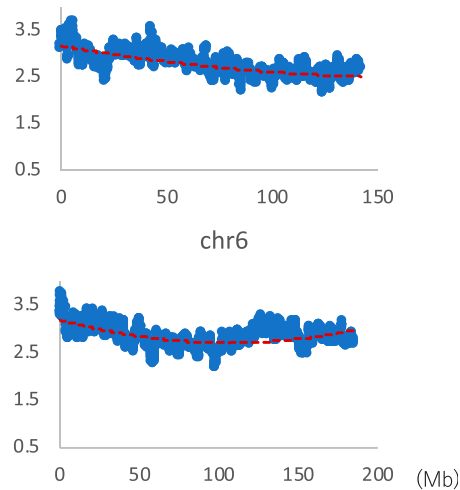

Figure 2. Distribution of CG composition in 1-Mb windows sliding with a 100-kb step on six chromosomes of four frogs.

(A) Pyxicephalus adspersus. (B) Xenopus tropicalis. (C) Xenopus laevis. (D) Leptobrachium leishanense. The binomial approximation is shown as a dashed line. In the graphs the $x$-axe shows positions in each chromosome (Mb), and the $y$-axe shows CG composition (\%).

evident for P. adspersus (Fig 2A), and the biological significance of the internal peaks, which are often located close to the central area, will be discussed later in connection with centromeric and pericentromeric heterochromatins.

CG distributions on all other chromosomes are presented in Fig S4A-D. In P. adspersus, downward parabolic patterns are observed for all autosomes, and chrZ has higher values in a relatively large internal area (Fig S4A). A distinct peak is observed at an internal position, often near the central area, of all chromosomes except chrW. In X. tropicalis and $X$. laevis, the same patterns are also observed for all 10 and 18 chromosomes, respectively, but a sharp decrease is seen very near the ends of all $X$. laevis chromosomes. For L. leishanense, with the lowest CG suppression at all chromosomes, downward parabolic patterns are observed for most chromosomes, but they tend to be weaker than for the other three frogs. 


\section{Variation of CG suppression indexes along chromosomes}

The variation of CG occurrences along the chromosome is inevitably influenced by variation in mononucleotide occurrences. Next, we examine whether the trend of increasing the frequency of CG toward both ends of chromosomes, as well as internal peaks that are prominent for $P$. adspersus, is a mere reflection of $\mathrm{G}+\mathrm{C} \%$ variation along chromosomes. Assuming that the $\mathrm{CG}$ variation is only the reflection of $\mathrm{G}+\mathrm{C} \%$ variation, the $\mathrm{CG} / \mathrm{GC}$ ratio should fluctuate around 1.0 on the chromosome. Fig 3 plots two indexes of CG suppression, the CG/GC ratio (orange) and the Obs/Exp ratio of CG (blue), for $P$. adspersus, which has the most distinct parabolic distribution. Both indexes are clearly lower than 1.0 over the entire chromosome. The CG variation is therefore not reflected by the $\mathrm{G}+\mathrm{C}$ $\%$ variation.

\section{Analysis of trinucleotide compositions}

Species-specific genome signatures become more pronounced as oligonucleotides become longer than dinucleotides (Abe et al, 2003, 2006). Fig $4 \mathrm{Ai}$ is a BLSOM for trinucleotide compositions, and its selforganization according to species becomes clearer than that for dinucleotide compositions. In Fig 4B, the degree of contribution of each trinucleotide to each node is displayed for eight examples; results of all trinucleotides are presented in Fig S5. The CGcontaining trinucleotides ( $\mathrm{ACG}+\mathrm{CGT}, \mathrm{CCG}+\mathrm{CGG}, \mathrm{CGA}+\mathrm{TCG}$, and $\mathrm{CGC}+\mathrm{GCG}$ ) occur more frequently (pink) in L. leishanense and $G$. rugosa than in other species, but, depending on the nucleotide added to $C G$, somewhat differential effects are observed for $G$. rugosa (Fig 4B, upper maps). The lower panels of Fig 4B show that three trinucleotides occur at low frequencies (green) in L. leishanense, but TCA+TGA appears at a higher frequency in G. rugosa than L. leishanense.

\section{Characterization of satellite territories of $P$. adspersus and $X$. laevis}

On the dinucleotide BLSOM (Fig 1Bii), tiny territories of a particular species often scatter in large territories of other species, but they tend to be grouped into a small number of species-specific satellite territories on the trinucleotide BLSOM (Fig 4Ai). For P. adspersus and $X$. laevis, there are a few small satellites (e.g., those arrowed in Fig 4Aii) far away from their main territories, and three satellites of P. adspersus (Psz1, Psz2, and Psz3 arrowed in Fig 4Aii) are in contact with the large G. rugosa territory (green in Fig 4Ai). To characterize these satellites at the sequence level, we obtained sequences belonging to each satellite (Table S1) and then calculated their trinucleotide compositions and its ratio to that of its entire genome. The ratios of the top 10 trinucleotides for Psz 1 are shown by red bars in Fig 4Aiii. The type of the top 10 trinucleotides for Psz2 (green) was the same as that for Psz1, but there was one difference for Psz3 (violet), as described in the legend of Fig 4Aiii. Considering their sequence types, not only the CG-containing trinucleotides but also the polypurine/polypyrimidine type (e.g., $C C C+G G G, C T C+G A G$, and $\mathrm{GGA}+\mathrm{TCC}$ ) also ranks high. The enrichment of the characteristic trinucleotides is likely a factor that creates satellite territories, and their differential abundance is probably a factor in creating different satellites.

For the $P$. adspersus chromosomes, Fig 5 analyzed the distribution of CCG+CGG, whose frequency is the highest in Psz1 and Psz2
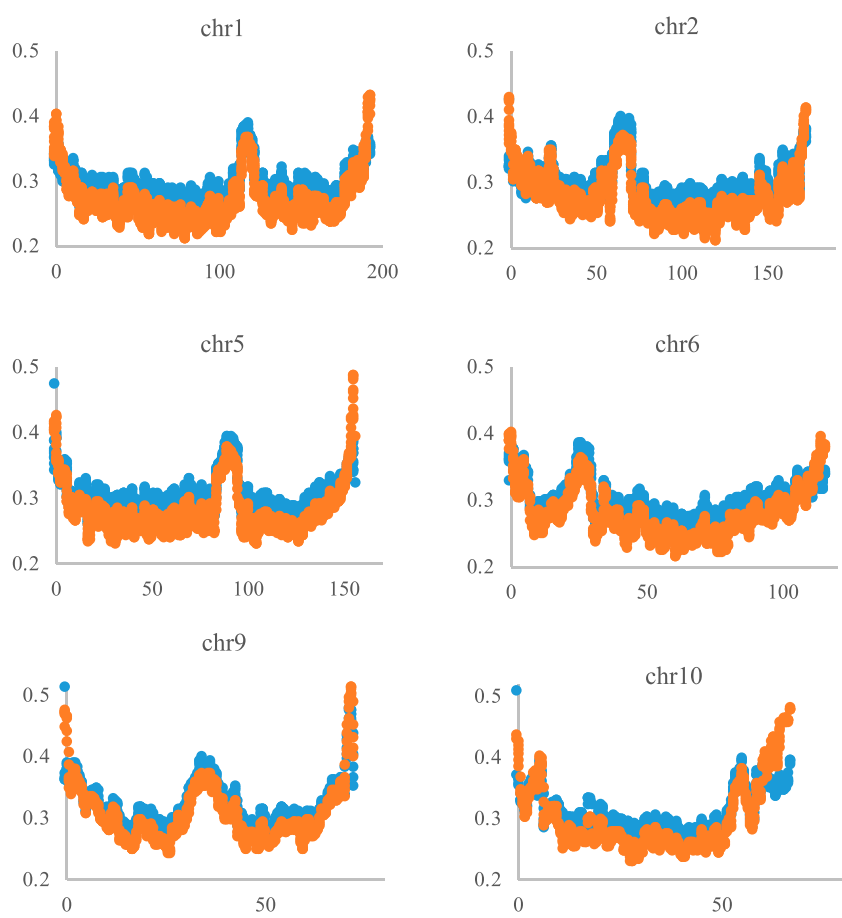
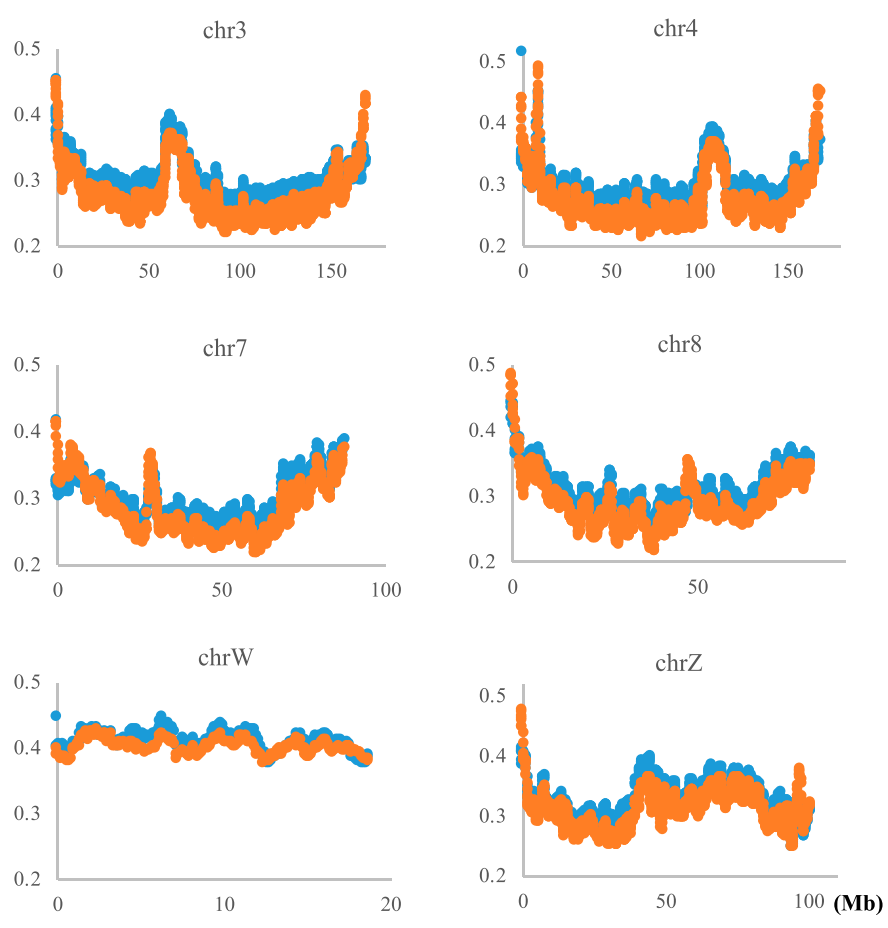

Figure 3. Distribution of the normalized CG (blue) and the CG/GC ratios (orange) on the Pyxicephalus adspersus chromosomes. 


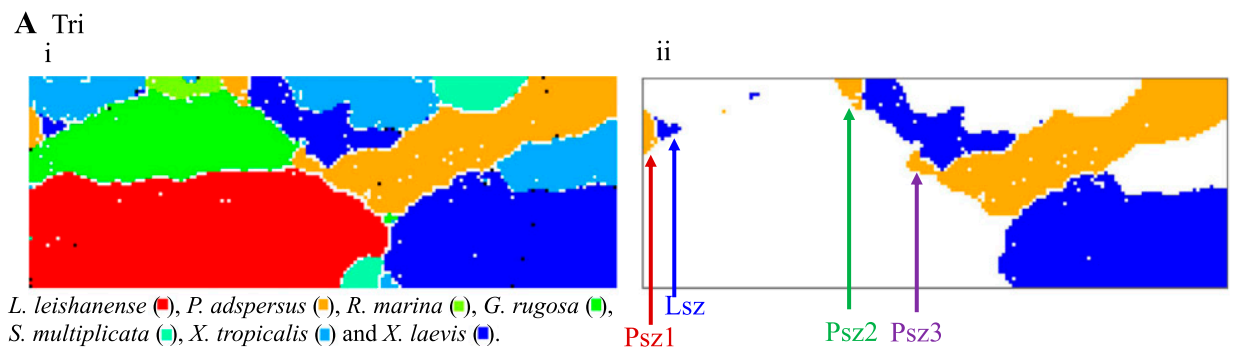

iii

B
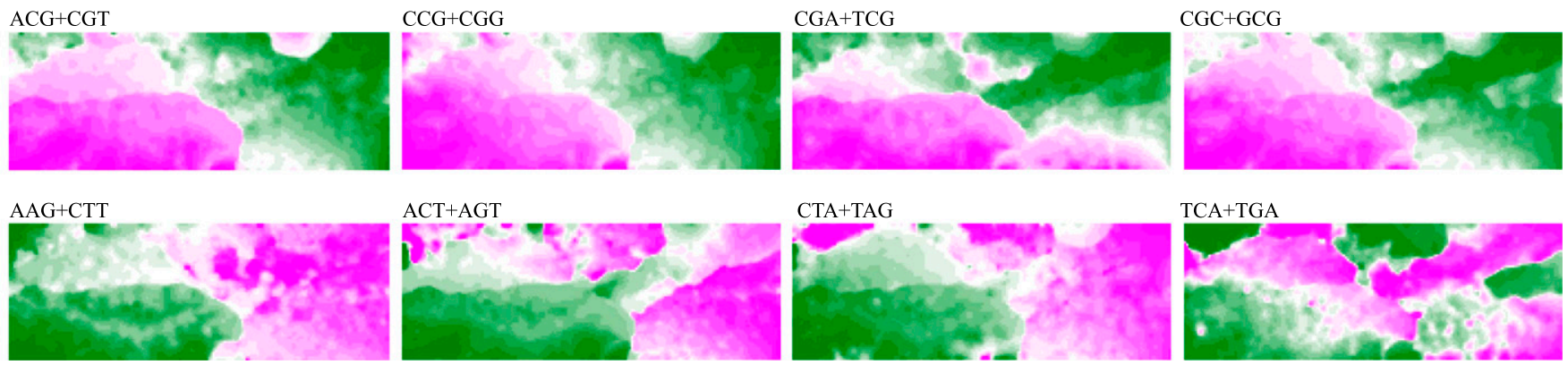

Figure 4. Trinucleotide (Tri) batch-learning self-organizing maps for 1-Mb sequences sliding with a 100-kb step.

(Ai) Nodes are marked as described in Fig 1Ai. (Aii) Sequences of Pyxicephalus adspersus and Xenopus laevis are displayed on the map, and their small satellite territories are marked by arrows. (Aiii) The ratio of occurrence of each of 10 trinucleotides in each satellite of $P$. adspersus to that in the entire genome is presented by a vertical colored bar: red (Psz1), green (Psz2), and violet (Psz3). Trinucleotides are arranged in the descending order of the ratio in Psz1, Psz2, and Psz3. For the Psz3 satellite, $A G G+C C T$ was included in its top 10 instead of ACC+GGT, but the result of ACC+GGT is presented for comparison with other satellites. (B) The contribution level of each trinucleotide to each node on the map is visualized as described in Fig 1C; results of all trinucleotides are presented in Fig S6.

and the second highest in Psz3. Similar to the CG distribution (Figs 1 and 2), the highest peak locates at or near the ends of all chromosomes except chrw. On and above the horizontal axis, chromosomal locations of sequences belonging to Psz1, Psz2 and Psz3 are indicated by red, green and violet marks, respectively. Psz1 sequences (red) are present on all chromosomes except chrw and mainly locate at the ends of most chromosomes. Psz2 sequences (green) also locate near the ends of most chromosomes but slightly inward from Psz1; on chrW, however, they widely scatter in internal areas. The Psz3 sequences (violet) are widely scattered across chrw and in multiple internal areas on chrz, but locate in an internal peak on chr1, chr2, chr3, chr6, and chr10 and at one end of chr8.

When the same analysis was performed on the small $X$. laevis territory (LSz in Fig 4Aii), the LSz sequences were found at and near the ends of all chromosomes except chr9_10S (data not shown). When the ratio of the trinucleotide frequency of these sequences to that of the entire $X$. laevis sequence was calculated, the value for polypurine/polypyrimidine-type trinucleotides, CCC+GGG and CTC+GAG, was higher than for those containing CG, showing an obvious difference from $P$. adspersus.

\section{G. rugosa and sex chromosomal sequences}

One reason for genome sequencing of $G$. rugosa is its intraspecies variability of sex chromosomal systems (Miura, 2007, 2017; Ogata et al, 2018). Using oligonucleotide BLSOMs, we next searched for $G$. rugosa sequences with a highly similar oligonucleotide composition to sex chromosomes of other frogs. On the trinucleotide BLSOM presented in Fig 4Ai, the species-dependent resolution was very high, and there were very few black nodes where G. rugosa sequences (green) overlap with those of other frogs, showing the resolution to be too high to identify sequences in search as black nodes. As a strategy for identifying the sequences with a highly similar oligonucleotide composition with sex chromosomes of other frogs, our previous method of sliding the 1-Mb window with 10-kb width (Wada et al, 2020) was considered to be suitable. In the BLSOM constructed for these sequence data, an average of 100 sequences per node was set (Fig 6). Even if one sequence of a certain species mixes with sequences of other species, the node is marked in black, and, therefore, overlapping of sequences with a highly similar composition among species will be detected as a black node. Furthermore, because of the very narrow sliding step, the composition in adjacent 1-Mb sequences becomes very similar, and sequences with a close genomic location should be visualized mainly as continuous dotted lines; that is, if there is a Mb-level structure with a highly similar composition between two species, this may be visualized as a black dotted line, rather than a single black dot. BLSOMs with di-, tri-, and tetranucleotide compositions are shown in $\mathrm{Fig} 6 \mathrm{Ai}, \mathrm{Bi}$, and $\mathrm{Ci}$, respectively. Then, using these BLSOMs, all sequences of $G$. rugosa (green) and sequences derived only from sex chromosomes of other frogs are displayed (Fig 6Aii, Bii, and Cii); as the sex chromosome, chrW (dark brown) and chrZ (light brown) have been reported for $P$. adspersus (Denton et al, 2018 Preprint), and chr7 of $X$. tropicalis (light blue) and chr2L of $X$. laevis (dark blue) have been reported (Session et al, 2016; Mitros et al, 2019).

For chrW of $P$. adspersus, its sequence occupies only small zones (dark brown in Fig 6Aii, Bii, and Cii) which locate mainly around the 

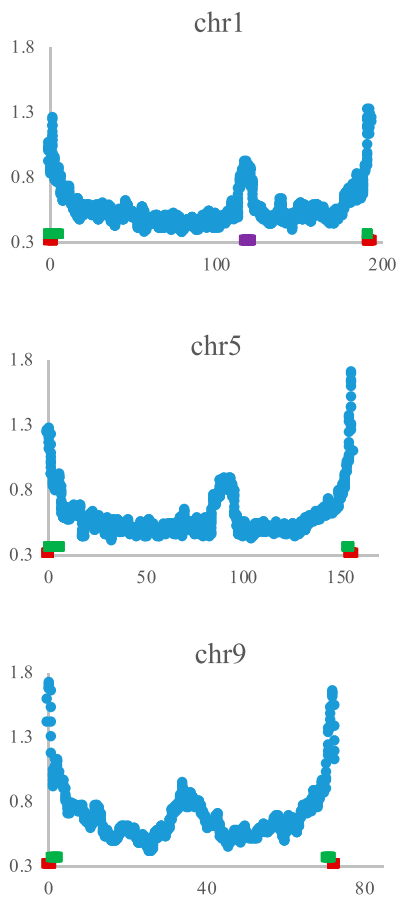
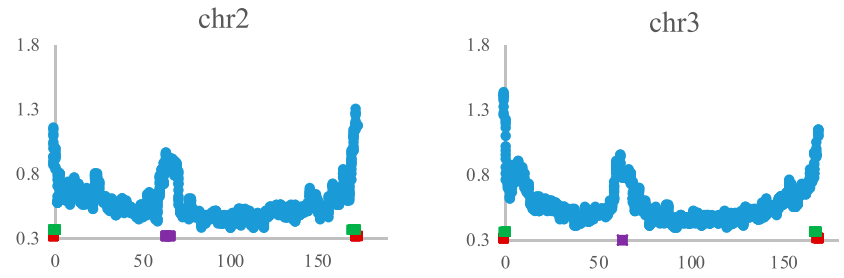

chr6
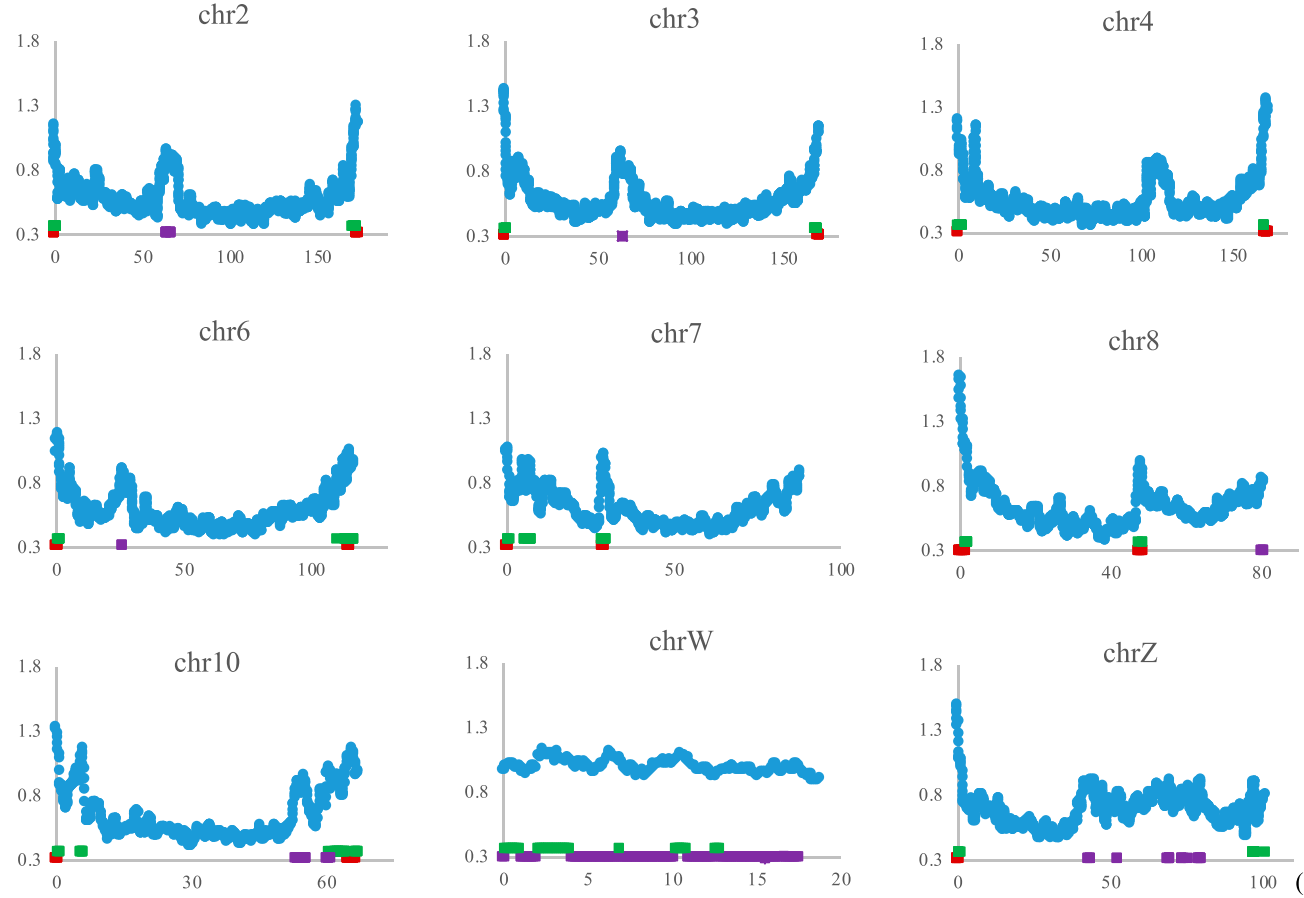

Figure 5. Distribution of CCG+CGG composition in 1-Mb windows sliding with a 100-kb step on the Pyxicephalus adspersus chromosomes.

Chromosomal locations of sequences for Psz1, Psz2, and Psz3 are indicated by thick horizontal lines in red, green and violet on and above the x-axis. In the graphs, the $\mathrm{x}$-axis shows positions in each chromosome (Mb), and the $\mathrm{y}$-axis shows CCG+CGG composition (\%).

G. rugosa territory. On the other hand, sequences of other sex chromosomes are widely distributed in the main territory of each species and are situated away from the G. rugosa territory. To precisely identify the overlap and proximity of G. rugosa and chrW in $P$. adspersus, all sequences of the two species are displayed (Fig 6Aiii, Biii, and Ciii). Di1 and D2 in Fig 6Aiii and Tri1 in Fig 6Biii show overlapped regions on di- and trinucleotide BLSOMs, respectively, and scaffold sequences of $G$. rugosa belonging to the black nodes were isolated. Although no overlap was seen on the tetranucleotide BLSOM, a series of chrW sequences were adjacent to the $G$. rugosa territory, as visualized as a dark-brown dotted line (Tet1 in Fig 6Cii), and the corresponding scaffold sequences were isolated.

\section{G. rugosa sequences with high sequence identity to chrW}

Of the isolated scaffold sequences from the three BLSOMs (Fig 6), four scaffold sequences of $G$. rugosa were commonly found in Di1, Tri1, and Tet1, and their sizes ranged from 1 to $2.7 \mathrm{Mb}$. To understand their overall similarity to chrW, a dot plot analysis (Cabanettes \& Klopp, 2018) was performed; two patterns are presented in Fig 7A and $\mathrm{B}$ and the other two are presented in Fig S6A and B. For each scaffold, dots locate mainly around 1.5, 4.5, 17, and $18 \mathrm{Mb}$ on chrW. For $17-\mathrm{Mb}$, dots with different levels of similarity are densely arranged across the entire area of each scaffold, but the actual density differs among scaffolds. As for other positions such as 18 $\mathrm{Mb}$, dots appear less densely and primarily at different positions from the 17-Mb case for each scaffold. Overall, these scaffolds are filled with repetitive elements with different levels of identity to the chrw sequence that is located mainly at four different positions; the length of these elements was around 1-3.5 kb. To identify actual sequences of the repetitive elements, we next performed a BLASTn search (https:/ / blast.ncbi.nlm.nih.gov/Blast.cgi?PAGE_TYPE=BlastSearch; Altschul et al, 1990) of the four scaffold sequences against the chrW sequence. The highest identity was $85 \%$ for $3.4 \mathrm{~kb}$, and many sequences with different levels of identity were found for each scaffold; examples of sequence alignments are presented in Supplemental Data 1-4.

A portion of the homologous sequences are $86-92 \%$ identical to a Bam transposon (520 bp) that was found only in the Ranidae family by the BLASTn search (Casola et al, 2004; Supplemental Data S5), and the Bam transposon is C/G-rich and similar to the large transposon element hAT (hobo-Activator-Tam3) superfamily (Ragghianti et al, 2004). The hAT occupied 5.9\% of the G. rugosa genome (Fig S7), and the Bam sequence has a high CG frequency (33\%). G. rugosa and P. adspersus are Ranidae family members, and a total of 250 scaffolds in the G. rugosa genome have 1,018 Bam copies (>82\% identity). P. adspersus has 75 Bam copies ( $>81 \%$ identity), and chrw includes four copies and are highly similar to the four scaffolds in G. rugosa as shown in the dot plots (Figs 7A and $B$ and S6A and B). The Bam sequences are thought to have increased in $G$. rugosa after its divergence from $P$. adspersus 89 million years ago (MYA) (http:/ / www.timetree.org), and the burst of transposons may have caused the increase in both size and CG composition in the whole $G$. rugosa genome.

Putting these observations together, G. rugosa has Mb-level structures rich in kb-level repetitive elements that are homologous to the chrw sequences (e.g., the Bam transposon), and this should provide fundamental knowledge for future studies of its sex chromosomes. On the BLSOMs displayed in Fig 5, there are 

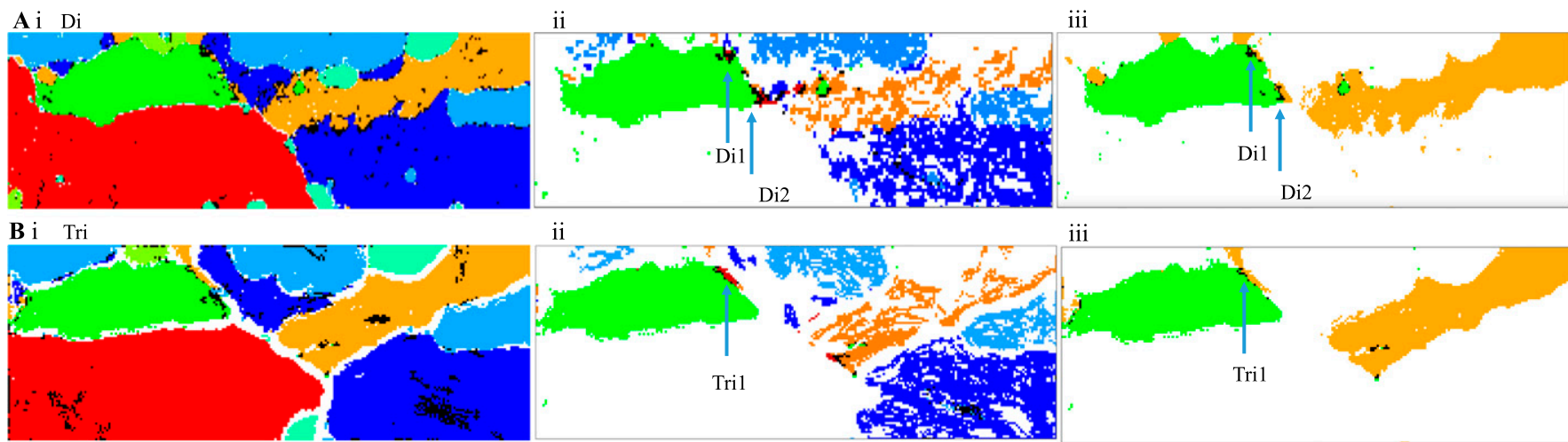

iii
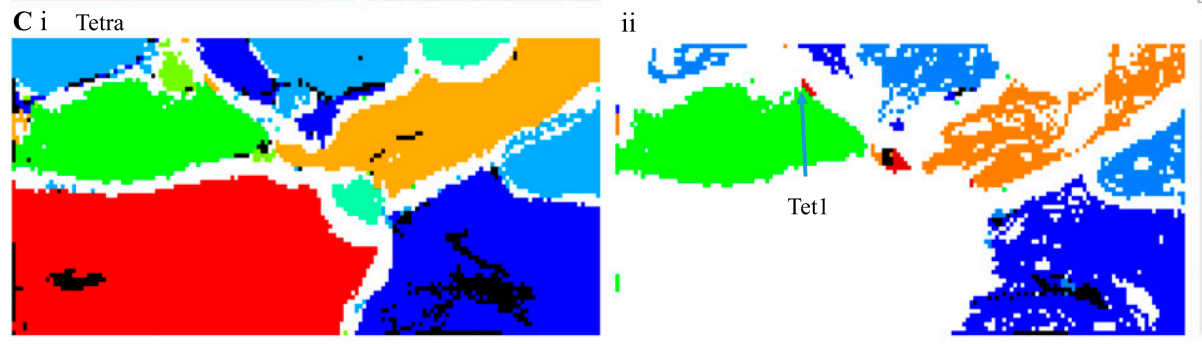

iii
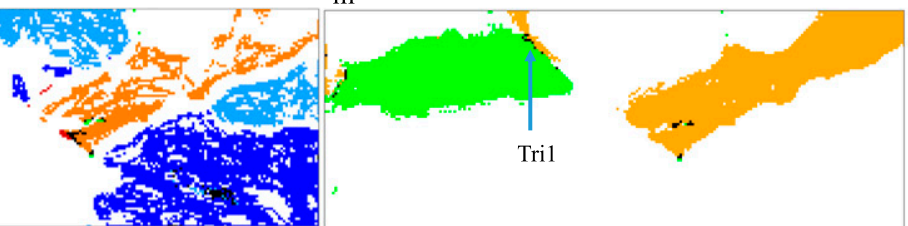

iii

leishanense (), P. adspersus (), R. marina (), G. rugosa (), P. Adspersus chrW (), chrW (() S. multiplicata ()$, X$. tropicalis () and X. laevis ( () .

Figure 6. Batch-learning self-organizing maps (BLSOMs) for 1-Mb sequences sliding with a 10-kb step.

(Ai, Bi, Ci) Di-, tri-, and tetranucleotide (Tetra) BLSOMs, respectively. (Aii, Bii, Cii) All sequences of Glandirana rugosa and sequences derived from sex chromosomes of other frogs are displayed: chrW (dark brown) and chrZ (light brown) of Pyxicephalus adspersus, chr7 of Xenopus tropicalis (light blue), and chr2L of Xenopus laevis (dark blue). Satellite territories of P. adspersus (dark brown) around the G. rugosa territory are marked by arrows. (Aiii, Biii, Ciii) All sequences of G. rugosa and P. adspersus are displayed. The black nodes containing both P. adspersus and G. rugosa sequences are specified by Di1 and Di2 on the di- and trinucleotide BLSOMs (Aiii and Biii, respectively), and sequences belonging to these black nodes were extracted. The G. rugosa nodes in the vicinity of chrW are marked by dark brown (Tet1; Ciii) and sequences belonging to the nodes were extracted.

additional regions, where sex chromosomal sequences of different species are overlapping, and we are undertaking a separate detailed study for characterizing these sequences.

\section{Discussion}

As the strategy for this data-driven study, knowledge discovery was left to the unsupervised and explainable AI, BLSOM. Then, based on the obtained knowledge, successive detailed analyses were conducted. We next discuss the biological significance of the obtained findings.

\section{CG suppression}

The level of CG suppression is highly conserved among mammals but varies substantially among frogs (Figs 1D and S3); for example, even for $G$. rugosa and $P$. adspersus, the values are 0.45 and 0.30 , respectively. G. rugosa and $P$. adspersus are diploid species and evolutionarily close to each other, but their assembled genome sizes differ greatly: G. rugosa, $7.08 \mathrm{~Gb}$ (this study), and P. adspersus (1.56 Gb: Denton et al, 2018 Preprint). The difference in the CG suppression may relate to the difference in genome sizes because numerous transcripts potentially present in large genomes should be severely suppressed in a very wide range of the genome, and their chromatin should therefore be highly condensed and heterochromatinized Grewal \& Jia, 2007). When C in CG undergoes methylation, the methylated CG tends to mutate to TG/CA, resulting in the CG suppression (i.e., CG deficiency) (Klose et al, 2005). However, if proteins bind stably to the DNA, the mutation is suppressed, and therefore, in constitutive heterochromatin regions, CGs are well conserved, resulting in the weaker CG suppression (Klose et al, 2005; Bogdanović \& Veenstra, 2009). This explanation is only one hypothesis, and other mechanisms such as involvement of ncRNA may work in suppressing gene expression in a wide genomic area.

\section{Generality and exception}

When most chromosomes of a certain species have a certain common characteristic, focusing on the exceptional chromosome should provide valuable information about the chromosome. The CG frequency does not have high peaks near the ends of chrW but is maintained at a high level over the entire area (Fig 2A), and this exceptional feature resembles human chry (Wada et al, 2015, 2020) of which a large portion is composed of constitutive heterochromatin. This suggests that a large portion of chrw, as well as the four G. rugosa scaffold sequences mentioned above, is composed of constitutive heterochromatin.

In the CG distribution on frog chromosomes (Figs 2 and S4), downward parabolic patterns are observed for almost all chromosomes, with the evident exception of chrW. Taking together the 

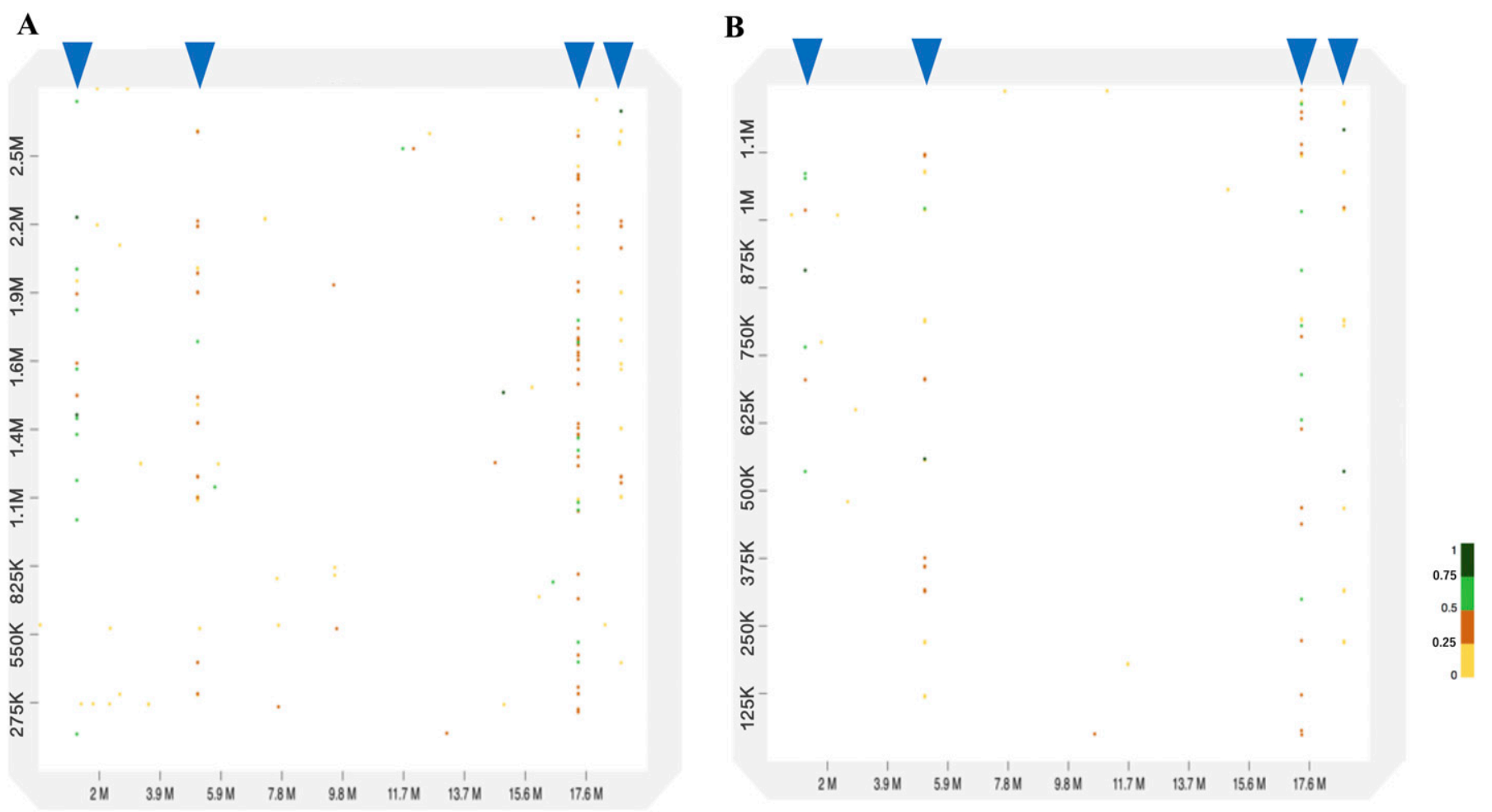

Figure 7. Dot plot analyses between chrW in Pyxicephalus adspersus and two scaffold sequences in Glandirana rugosa.

(A, B) Each $\mathrm{x}$-axis is the position of chrW, and the y axes are locations of (A) scaffold2393955 and (B) scaffold4079606. Blue arrowheads indicate the position of a series of dots in $1.5,4.5,17$, and $18 \mathrm{Mb}$ on chrW.

continuously changing parabolic pattern across each chromosome and the generality for all frogs, this characteristic is thought to relate to global features of chromosomal DNA segments, such as their nuclear locations: nuclear envelope side, internal side and so on. Signal sequences responsible for the nuclear organization may not be embedded in a few narrow chromosomal regions but distributed in broader ones (Bernardi, 2019).

In addition to the distribution of CG in Figs 2 and S4, Fig 5 shows the CCG+CGG distribution for almost all P. adspersus chromosomes, and therefore, exceptional cases can be easily explained. On all autosomes, the continuously changing parabolic pattern is seen, but in chr7 and chr8, somewhat discontinuous changes appear at an internal peak site (Figs 5 and S4). Furthermore, Psz1 and Psz2 sequences (red and green bars, respectively), which locate primarily at the ends of other autosomes, locate at the internal sites of chr7 and chr8; and Psz3 sequences (violet), which locate at the internal peak of other autosomes, locate at one end of chr8 (Fig 5). These irregularities may reflect an intra- or interchromosomal rearrangement, such as via the telomere and centromere.

\section{Similarity of Mb-level peaks of frogs and human}

This study mainly analyzed CG dinucleotide and CG-containing trinucleotides, and found $\mathrm{Mb}$-level structures rich in these oligonucleotides. We next discuss the internal peaks most evidently observed on the P. adspersus chromosomes. Our previous Mb-level analyses on the human chromosomes found high CG peaks (a Mb- level CpG island) in centromeric and pericentromeric constitutive heterochromatin regions of all chromosomes except chrY (Wada et al, 2015, 2020). Those studies also analyzed oligonucleotides longer than pentanucleotides and found that a wide range of transcription factor binding sequences (TFBSs) were enriched in $\mathrm{Mb}$ level CpG islands located in the centromeric and pericentromeric regions (Iwasaki et al, 2013; Wada et al, 2020); specifically, we analyzed a total of $~ 5,000$ TFBSs of hexa-to octanucleotides compiled by the SwissRegulon Portal (Pachkov et al, 2013) and found that the enriched TFBS types differ depending on the chromosome.

The present frog genome study focused on mono- to tetranucleotides, and TFBS occurrences cannot be directly discussed. For longer oligonucleotides, we have been conducting a separate detailed study but have preliminarily analyzed eight pentanucleotides that are consensus core elements for a wide variety of vertebrate TFBSs (Iwasaki et al, 2013; Fornes et al, 2020) and found that the TFBS core elements, such as GATA-containing or polypurine/polypyrimidine-type pentanucleotides, are evidently enriched in the internal Mb-level CpG islands of $P$. adspersus (Fig S7). When considering similarity to the human chromosomes, the internal $\mathrm{Mb}$-level $\mathrm{CpG}$ islands of $\mathrm{P}$. adspersus are surmised to correspond to the centromeric and pericentromeric heterochromatin regions enriched in TFBSs.

If this idea is correct, it would seem contradictory that there are few internal Mb-level CpG islands on the X. laevis chromosomes (Fig 2). An additional analysis on its chromosomes for the above TFBS cores showed a distinct internal peak which mainly locates close to 
the central area of each chromosome and is enriched for the TFBS cores such as a polypurine/polypyrimidine-type pentanucleotide, CTTCC+GGAAG (Fig S8). In the case of X. laevis, CG occurrences are ubiquitously higher than for $P$. adspersus, and, therefore, various TFBSs, rather than CG-containing oligonucleotides, may be specifically enriched in its centromeric and pericentromeric heterochromatin regions as clearly marking the functionally important structure.

\section{Perspectives on the evolution of sex chromosomes}

Sex chromosomes are not well developed in the frogs analyzed here, except in P. adspersus which has 12 autosomes and a pair of heteromorphic sex (ZW) chromosomes (Denton et al, 2018 Preprint). G. rugosa has 13 chromosomes, and chr7 is a homomorphic sex chromosome (Miura, 2017). The sex chromosomes in these two frogs are believed to have diverged at least 89 MYA, and they have three common sex chromosomal genes: SRY-box 3 (SOX3), $\alpha$ thalassemia / mental retardation syndrome $X$-linked (ATRX), and androgen receptor (Miura, 2017; Denton et al, 2018 Preprint). These genes are candidates for a sex-determining gene in $G$. rugosa and $P$. adspersus and are also located on the human sex chromosomes. SOX3 is an X-linked homologous gene of a sex-determining region $Y$ (SRY) gene which is a therian male determiner and diverged from SOX3 160 MYA (Katsura et al, 2018). In addition, ATRX is located on the sex chromosome in the Mexican axolotl (Ambystoma mexicanum), and the W-linked gene (ATRW) is a candidate of the sexdetermining gene (Keinath et al, 2018). These observations suggest the convergent molecular evolution that some similar characteristics have been obtained on the sex chromosomes in therians and amphibians independently.

Sex-determining systems differ among frogs. In X. laevis, a female determiner, the $\mathrm{W}$-linked double-sex and mab-3 (DM) domain gene (Dm-W), has been identified (Yoshimoto et al, 2008). Dm-W was duplicated from the DM related transcription factor 1 gene (DMRT-1), and DMRT-1 is known to be a master sex-determining gene in several vertebrates (Bachtrog et al, 2014; Miura, 2017). In G. rugosa and $P$. adspersus, however, DMRT-1 is on an autosome and is probably not the sex-determining gene. The Mb-level structures of $G$. rugosa that are rich in kb-level repetitive elements homologous to chrW sequences of $P$. adspersus should provide fundamental knowledge for future studies of sex chromosomes of these species.

\section{Perspectives on BLSOM analyses of comparative genomics}

In the present study, we focused on mono-to tetranucleotide compositions primarily in 1-Mb fragments and conducted comparative genome analyses using different species. Here, we discuss its usefulness for characterizing an intraspecies difference. As a preliminary analysis, we examined whether the large and small chromosomes of $X$. laevis, which were derived from a heteroploid of two different species (Session et al, 2016), were separated on the tetranucleotide-BLSOM presented in Fig 6C. Whereas the separation between large and small chromosomes of $X$. laevis was poor in its major territories, the sequences that invaded other species' territories showed clear differences between the large and small chromosomes; for example, sequences that invaded the $P$. adspersus territory were derived primarily from the large chromosome of X. laevis (Fig S9). This indicates usefulness of the BLSOM analysis for identifying intraspecies differences.

The present study showed that the genome signature was different among frogs and that the oligonucleotide compositions on chrY and chrw were similar beyond species. In future, details of the genome and sex chromosomal evolution will be better described by various BLSOM tools after the completion of detailed sequencing of the $G$. rugosa genome.

\section{Materials and Methods}

\section{Genome sequencing and de novo assembly}

We extracted DNA from a $G$. rugosa male (ZZ) individual collected in Kyoto (Ogata et al, 2018). One paired-end library (insert size, 300) and four mate-pair libraries (insert size, 3,000, 6,000, 10,000, and 15,000) were prepared using a TruSeq DNA PCR-Free LT Sample Prep Kit and a Nextera Mate Pair Sample Prep Kit, respectively. Sequencing was performed using Illumina HiSeq 2500 and NovaSeq 6000 (Table S2). Adapters and low-quality regions in reads were trimmed using Platanus_trim 1.0.7 (http:// platanus.bio.titech.ac.jp/ pltanus_trim) with the default parameters. The assembled genome size and heterozygosity were estimated by GenomeScope software (Vurture et al, 2017) after inputting the trimmed paired-end reads, and the estimated coverage depth was 154 (paired-ends, 117; matepairs, 37). De novo assembly was performed using Platanus-allee 2.2.2 (Kajitani et al, 2019) with the default parameters except for those related to multi-threading (-t 80 ) and maximum memoryusage (-m 2048). Note that only the paired-end library was input into the contig-assembly ("platanus_allee assmble" command), whereas all libraries were used for the following steps ("platanus_allee phase" and "platanus_allee consensus" commands). The sequence of the mitochondrial genome was separately constructed by a manual curation. Finally, short sequences $(<500$ bp) were discarded from the consensus (haploid) scaffolds set, and fragmented mitochondrial sequences were replaced using the BLASTn search (Altschul et al, 1990).

We detected the repetitive sequences using RepeatModeler2 (Flynn et al, 2020) and RepeatMasker (Smit et al, 2013-2015; http:// www.repeatmasker.org), and the occurrence ratios of each repetitive sequence to the whole genome are shown in Fig S10.

\section{BLSOM}

A self-organizing map (SOM) is an unsupervised algorithm that projects high-dimensional data nonlinearly onto a twodimensional plane (Kohonen et al, 1996), and Kanaya et al (2001) modified Kohonen's SOM for genome informatics to make the learning process and resulting map independent on the order of data input on the basis of a batch-learning SOM. In Kohonen's original SOM, the initial vectorial data were set by random values, but in BLSOM, the initial vectors were set based on the widest scale of the sequence distribution in the oligonucleotide frequency space with principal component analysis. Weights in the first 
dimension (I) were arranged into lattices corresponding to a width of five times the SD (501) of the first principal component: the second dimension ( $J$ ) was defined by the nearest integer greater than $\sigma 2 / \sigma 1 \times 1$; and I was the average number of sequence data per node. $\sigma 1$ and $\sigma 2$ were the standard deviations of the first and second principal components, respectively. The weight vector on the $i j^{\text {th }}$ lattice $\left(w_{i j}\right)$ was represented as follows ( $i$ and $j$ represent the position of lattice points):

$$
W_{i, j}=x_{a v}+\frac{5 \sigma_{1}}{l}\left[b_{1}\left(i-\frac{l}{2}\right)+b_{2}\left(j-\frac{1}{2}\right)\right] \text {, }
$$

where $x_{a v}$ is the average vector for oligonucleotide frequencies of all input vectors, and $b_{1}$ and $b_{2}$ are eigenvectors for the first and second principal components. Weight vectors $\left(w_{i j}\right)$ were set and updated as described previously (Abe et al, 2003).

Because principal component analysis can grasp basic properties of genomic sequences such as $\mathrm{G}+\mathrm{C} \%$, the global patterns of oligonucleotide BLSOMs, in which various learning parameters and the number of sequences per node are changed, resemble each other (Abe et al, 2003, 2005). The BLSOM for oligonucleotide composition was constructed as described by Abe et al (2003), and oligonucleotides diagnostic for category-dependent separation were visualized as described by Kanaya et al (2001). The BLSOM program can be obtained from a GitHub repository (https:/ / github.com/ yukakokatsura/BLSOM).

\section{Data Availability}

The DNA read libraries and the genome assembly of G. rugosa have been deposited at the DNA Data Bank of Japan (DDBJ) Sequence Read Archive (DRA009996) under BioProject PRJDB9666. Genome sequences of six frogs were obtained from the following ftp sites and accession numbers: L. leishanense (https://ftp.ncbi.nlm.nih.gov/genomes/all/GCA/ 009/667/805/GCA_009667805.1_ASM966780v1/), P. adspersus (CM016416CM016429), X. tropicalis (http://ftp.xenbase.org/pub/Genomics/JGI/ Xentr10.0/), X. laevis (http://ftp.xenbase.org/pub/Genomics/JGI/ Xenla9.2/), R. marina (ftp://parrot.genomics.cn/gigadb/pub/10.5524/ 100001_101000/100483/canetoad.v2.2.fasta.gz) and S. multiplicata (VKOC01000001-VKOC01049736).

\section{Supplementary Information}

Supplementary Information is available at https://doi.org/10.26508/lsa. 202000905.

\section{Acknowledgements}

We thank Dr. Yohey Terai for helping with the purification of DNA. This work was supported by Japan Society for the Promotion of Science (JSPS) KAKENHI grants $16 \mathrm{H} 06279$ (Platform for Advanced Genome Science [PAGS]), 18 K14766 (Grant-in-Aid for Early-Career Scientists), and the Ministry of Education, Culture, Sports, Science and Technology (MEXT) Program for the Development of Next-generation Leading Scientists with Global Insight (L-INSIGHT).

\section{Author Contributions}

Y Katsura: conceptualization, resources, data curation, formal analysis, supervision, funding acquisition, validation, investigation, visualization, methodology, project administration, and writing-original draft, review, and editing.

T Ikemura: data curation, formal analysis, supervision, validation, investigation, visualization, methodology, project administration, and writing-original draft, review, and editing.

R Kajitani: resources, data curation, software, formal analysis, validation, investigation, visualization, methodology, and writingoriginal draft.

A Toyoda: resources, data curation, formal analysis, validation, investigation, methodology, and writing-original draft.

T Itoh: data curation, software, supervision, validation, and methodology.

M Ogata: resources.

I Miura: resources and writing-review and editing.

K Wada: software.

Y Wada: software.

Y Satta: conceptualization and writing-original draft, review, and editing.

\section{Conflict of Interest Statement}

The authors declare that they have no conflict of interest.

\section{References}

Abe T, Kanaya S, Kinouchi M, Ichiba Y, Kozuki T, Ikemura T (2003) Informatics for unveiling hidden genome signatures. Genome Res 13: 693-702. doi:10.1101/gr.634603

Abe T, Sugawara H, Kanaya S, Kinouchi M, Ikemura T (2006) Self-Organizing Map (SOM) unveils and visualizes hidden sequence characteristics of a wide range of eukaryote genomes. Gene 365: 27-34. doi:10.1016/ j.gene.2005.09.040

Abe T, Sugawara H, Kinouchi M, Kanaya S, Ikemura T (2005) Novel phylogenetic studies of genomic sequence fragments derived from uncultured microbe mixtures in environmental and clinical samples. DNA Res 12: 281-290. doi:10.1093/dnares/dsi015

Altschul SF, Gish W, Miller W, Myers EW, Lipman DJ (1990) Basic local alignment search tool. J Mol Biol 215: 403-410. doi:10.1016/s00222836(05)80360-2

Bachtrog D, Mank JE, Peichel CL, Kirkpatrick M, Otto SP, Ashman TL, Hahn MW, Kitano J, Mayrose I, Ming R, et al (2014) Sex determination: Why so many ways of doing it? PLoS Biol 12: e1001899. doi:10.1371/ journal.pbio.1001899

Bernardi G (2019) The genomic code: A pervasive encoding/molding of chromatin structures and a solution of the "non-coding DNA" mystery. BioEssays 41: 1900106. doi:10.1002/bies.201900106

Bogdanović O, Veenstra GJC (2009) DNA methylation and methyl-CpG binding proteins: Developmental requirements and function. Chromosoma 118: 549-565. doi:10.1007/s00412-009-0221-9

Cabanettes F, Klopp C (2018) D-GENIES: Dot plot large genomes in an interactive, efficient and simple way. PeerJ 6: e4958. doi:10.7717/ peerj. 4958

Casola C, Marracci S, Bucci S, Ragghianti M, Mancino G, Hotz H, Uzzell T, Guex GD (2004) A hAT-related family of interspersed repetitive elements in 
genomes of western Palaearctic water frogs. J Zool Syst Evol Res 42: 234-244. doi:10.1111/j.1439-0469.2004.00254.x

Denton RD, Kudra RS, Malcom JW, Du Preez L, Malone JH (2018) The African Bullfrog (Pyxicephalus adspersus) genome unites the two ancestral ingredients for making vertebrate sex chromosomes. BioRxiv doi:10.1101/329847Preprint posted November 20, 2018.

Edwards RJ, Tuipulotu DE, Amos TG, O'Meally D, Richardson MF, Russell TL, Vallinoto M, Carneiro M, Ferrand N, Wilkins MR, et al (2018) Draft genome assembly of the invasive cane toad, Rhinella marina. Gigascience 7: giy095. doi:10.1093/gigascience/giy095

Flynn JM, Hubley R, Goubert C, Rosen J, Clark AG, Feschotte C, Smit AF (2020) RepeatModeler2 for automated genomic discovery of transposable element families. Proc Natl Acad Sci U S A 17: 9451-9457. doi:10.1073/ pnas. 1921046117

Fornes O, Castro-Mondragon JA, Khan A, van der Lee R, Zhang X, Richmond PA, Modi BP, Correard S, Gheorghe M, Baranašić D, et al (2020) JASPAR 2020: Update of the open-access database of transcription factor binding profiles. Nucleic Acids Res 48: D87-D92. doi:10.1093/nar/ gkz1001

Grewal S, Jia S (2007) Heterochromatin revisited. Nat Rev Genet 8: 35-46. doi:10.1038/nrg2008

Hellsten U, Harland RM, Gilchrist MJ, Hendrix D, Jurka J, Kapitonov V, Ovcharenko I, Nicholas H, Putnam NH, Shu S, et al (2010) The genome of the western clawed frog Xenopus tropicalis. Science 328: 633-636. doi:10.1126/science. 1183670

Iwasaki Y, Abe T, Okada N, Wada K, Wada Y, Ikemura T (2014) Evolutionary changes in vertebrate genome signatures with special focus on coelacanth. DNA Res 21: 459-467. doi:10.1093/dnares/dsu012

Iwasaki Y, Wada K, Wada Y, Abe T, Ikemura T (2013) Notable clustering of transcription-factor-binding motifs in human pericentric regions and its biological significance. Chromosome Res 21: 461-474. doi:10.1007/ s10577-013-9371-y

Kajitani R, Yoshimura D, Okuno M, Minakuchi Y, Kagoshima H, Fujiyama A, Kubokawa K, Kohara Y, Toyoda A, Itoh T (2019) Platanus-allee is a de novo haplotype assembler enabling a comprehensive access to divergent heterozygous regions. Nat Commun 10: 1702. doi:10.1038/ s41467-019-09575-2

Kanaya S, Kinouchi M, Abe T, Kudo Y, Yamada Y, Nishi T, Mori H, Ikemura T (2001) Analysis of codon usage diversity of bacterial genes with a selforganizing map (SOM): Characterization of horizontally transferred genes with emphasis on the E. coli 0157 genome. Gene 276: 89-99. doi:10.1016/s0378-1119(01)00673-4

Karlin S (1998) Global dinucleotide signatures and analysis of genomic heterogeneity. Curr Opin Microbiol 1: 598-610. doi:10.1016/s13695274(98)80095-7

Katsura Y, Kondo HX, Ryan J, Harley V, Satta Y (2018) The evolutionary process of mammalian sex determination genes focusing on marsupial SRYS. BMC Evol Biol 18: 3. doi:10.1186/s12862-018-1119-z

Keinath MC, Timoshevskaya N, Timoshevskiy VA, Voss SR, Smith JJ (2018) Miniscule differences between sex chromosomes in the giant genome of a salamander. Sci Rep 8: 17882. doi:10.1038/s41598-018-36209-2

Klose RJ, Sarraf SA, Schmiedeberg L, McDermott SM, Stancheva I, Bird AP (2005) DNA binding selectivity of MeCP2 due to a requirement for $A / T$ sequences adjacent to methyl-CpG. Mol Cell 19: 667-678. doi:10.1016/ j.molcel.2005.07.021

Kohonen T, Oja E, Simula O, Visa A, Kangas J (1996) Engineering applications of the self-organizing map. Proc IEEE 84: 1358-1384. doi:10.1109/ 5.537105

Law JA, Jacobsen SE (2010) Establishing, maintaining and modifying DNA methylation patterns in plants and animals. Nat Rev Genet 11: 204-220. doi:10.1038/nrg2719
Li J, Yu H, Wang W, Fu C, Zhang W, Han F, Wu H (2019) Genomic and transcriptomic insights into molecular basis of sexually dimorphic nuptial spines in Leptobrachium leishanense. Nat Commun 10: 5551. doi:10.1038/s41467-019-13531-5

Mitros T, Lyons JB, Session AM, Jenkins J, Shu S, Kwon T, Lane M, Ng C, Grammer TC, Khokha MK, et al (2019) A chromosome-scale genome assembly and dense genetic map for Xenopus tropicalis. Dev Biol 452: 8-20. doi:10.1016/j.ydbio.2019.03.015

Miura I (2007) An evolutionary witness: The frog rana rugosa underwent change of heterogametic sex from XY male to ZW female. Sex Dev 1 323-331. doi:10.1159/000111764

Miura I (2017) Sex determination and sex chromosomes in Amphibia. Sex Dev 11: 298-306. doi:10.1159/000485270

Nakao R, Abe T, Nijhof AM, Yamamoto S, Jongejan F, Ikemura T, Sugimoto C (2013) A novel approach, based on BLSOMs (Batch Learning SelfOrganizing Maps), to the microbiome analysis of ticks. ISME J 7: 1003-1015. doi:10.1038/ismej.2012.171

Ogata M, Lambert M, Ezaz T, Miura I (2018) Reconstruction of female heterogamety from admixture of XX-XY and ZZ-ZW sex-chromosome systems within a frog species. Mol Ecol 20: 4078-4089. doi:10.1111/ mec.14831

Pachkov M, Balwierz PJ, Arnold P, Ozonov E, van Nimwegen E (2013) SwissRegulon, a database of genome-wide annotations of regulatory sites: Recent updates. Nucleic Acids Res 41: D214-D220. doi:10.1093/ nar/gks1145

Ragghianti M, Bucci S, Casola C, Marracci S, Mancino G (2004) Molecular investigations in western palearctic water frogs. Ital J Zool 71: 17-23. doi:10.1080/11250000409356601

Seidl F, Levis NA, Schell R, Pfennig DW, Pfennig KS, Ehrenreich IM (2019) Genome of spea multiplicata, a rapidly developing, phenotypically plastic, and desert-adapted spadefoot toad. G3 9: 3909-3919. doi:10.1534/g3.119.400705

Session AM, Uno Y, Kwon T, Chapman JA, Toyoda A, Takahashi S, Fukui A, Hikosaka A, Suzuki A, Kondo M, et al (2016) Genome evolution in the allotetraploid frog Xenopus laevis. Nature 538: 336-343. doi:10.1038/ nature19840

Smit AFA, Hubley R, Green P (2013-2015) RepeatMasker Open-4.0. http:/ / www.repeatmasker.org (Accessed 18 December, 2020).

Vurture GW, Sedlazeck FJ, Nattestad M, Underwood CJ, Fang H, Gurtowski J, Schatz MC (2017) GenomeScope: Fast reference-free genome profiling from short reads. Bioinformatics 33: 2202-2204. doi:10.1093/ bioinformatics/btx153

Wada Y, Iwasaki Y, Abe T, Wada K, Tooyama I, Ikemura T (2015) CG-containing oligonucleotides and transcription factor-binding motifs are enriched in human pericentric regions. Genes Genet Syst 90: 43-53. doi:10.1266/ggs.90.43

Wada Y, Wada K, Ikemura T (2020) Mb-level CpG and TFBS islands visualized by $\mathrm{Al}$ and their roles in the nuclear organization of the human genome. Genes Genet Syst 95: 29-41. doi:10.1266/ggs.19-00027

Yoshimoto S, Okada E, Umemoto H, Tamura K, Uno Y, Nishida-Umehara C, Matsuda Y, Takamatsu N, Shiba T, Ito M (2008) A W-linked DM-domain gene, DM-W, participates in primary ovary development in Xenopus laevis. Proc Natl Acad Sci U S A 105: 2469-2474. doi:10.1073/ pnas. 0712244105

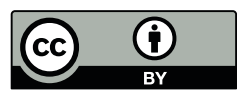

License: This article is available under a Creative Commons License (Attribution 4.0 International, as described at https://creativecommons.org/ licenses/by/4.0/). 